\title{
Clinical necessity of the immunohistochemical reassessment of para-aortic lymph nodes in resected pancreatic ductal adenocarcinoma
}

\author{
SUNG HOON CHOI ${ }^{1,2}$, SE HOON KIM ${ }^{3}$, JUN JEONG CHOI ${ }^{4}$, CHANG MOO KANG ${ }^{1,2}$, \\ HO KYOUNG HWANG ${ }^{1,2}$ and WOO JUNG LEE ${ }^{1,2}$
}

${ }^{1}$ Department of Surgery, Yonsei University College of Medicine; ${ }^{2}$ Pancreaticobiliary Cancer Clinic, Institute of Gastroenterology, Severance Hospital; ${ }^{3}$ Department of Pathology, Yonsei University College of Medicine, Seoul 120-752; ${ }^{4}$ Department of Pathology, Yonsei University Wonju College of Medicine, Wonju 220-050, South Korea

Received March 7, 2013; Accepted July 3, 2013

DOI: $10.3892 / 01.2013 .1539$

\begin{abstract}
Para-aortic lymph node (PALN) metastasis is widely regarded as a systemic disease in cancer. Undetected PALN micrometastases during routine hematoxylin and eosin (HE) staining may be a cause of poor prognosis following a potentially curative pancreatectomy for pancreatic cancer. In the present study, paraffin-embedded PALN tissue blocks from 99 patients who underwent a pancreatectomy were re-evaluated by immunohistochemical staining using cytokeratin (CK)-19. Patients with PALN metastasis were summarized according to the clinicopathological data. A total of 484 PALNs (median, 4.9 nodes per patient; range, 1-19) were evaluated. PALN metastases were revealed in eight patients $(8.1 \%)$ by routine HE staining of frozen section biopsies and in one patient (1.0\%) by HE staining of a permanent section. Only one patient (1.0\%) demonstrated micrometastasis by IHC; this patient did not display any adverse pathological characteristics and had a relatively favorable survival period of 41 months. The present study concluded that an additional reassessment for micrometastasis in PALNs using CK-19 immunohistochemistry (IHC) is not a viable method for determining the survival outcome. A careful examination of a frozen section biopsy is sufficient for attempting curative surgery.
\end{abstract}

\section{Introduction}

Surgical resection is the only known curative option for pancreatic cancer. However, the majority of pancreatic cancers

Correspondence to: Dr Chang Moo Kang, Department of Surgery, Yonsei University College of Medicine, Ludlow Faculty Research Building no. 204, 50 Yonsei-ro, Seodaemun-gu, Seoul 120-752, South Korea

E-mail: cmkang@yuhs.ac

Key words: pancreatic cancer, paraaortic lymph node, micrometastasis, immunohistochemistry are usually diagnosed at advanced stages and only $15-20 \%$ of patients are candidates for a gross margin-negative pancreatectomy (R0) (1). Following a curative resection, distant metastases, particularly in the liver, local recurrence and peritoneal dissemination frequently occur and these patients succumb to their diseases $(2,3)$ The reported five-year survival rate following surgical resection is $12.1-25.0 \%$ (1-5) and the overall survival rate is considered to be $<5 \%$, suggesting that pancreatic cancer is one of the most lethal gastrointestinal malignancies.

According to a previous survival analysis of resected pancreatic cancers, an R0 was shown to be an independent favorable prognostic factor (6-8). Over the past few decades, surgeons have attempted to achieve ideal R0 resections by extending the surgical margins in the hopes that clearing the surrounding soft tissue that contains malignant cells may improve the survival outcome. However, several significant prospective randomized control studies revealed that extending the margins did not result in an additional survival benefit over the standard surgery in resectable pancreatic cancer (9-12). It has been proposed that a possible underestimation of microscopic cancer spreading beyond the surgical field may contribute to a poor prognosis following a potentially curative surgical treatment (13).

Lymph node metastasis is common in pancreatic cancer (14-16) and para-aortic lymph nodes (PALNs) are considered to be the final nodes in the systemic lymphatic circulation in periampullary cancer $(17,18)$. Although there have only been a few studies on the oncological outcome according to PALN staging (19-21), PALN involvement is known to be a poor prognostic factor in periampullary tumors (18). However, pancreatic surgeons may encounter clinical cases of potentially resectable pancreatic tumors with unexpected PALN metastasis that are only identified on intraoperative frozen section biopsies. Considering the expected poor prognosis in patients with unexpected PALN metastasis and the potential curative role of an R0 in pancreatic cancer, the decision to resect must be promptly determined in the operating theatre.

The present study aimed to develop an approach to this clinical dilemma. The oncological outcomes in patients with 
PALN metastasis that were detected by hematoxylin and eosin (HE) staining were analyzed in resected pancreatic tumors. Immunohistochemistry (IHC) with antibodies against cytokeratin (CK)-19 was used to detect the presence of PALN micrometastasis in resected pancreatic ductal adenocarcinoma. The role of curative surgery in resectable pancreatic cancer with incidentally identified PALN metastasis was further investigated using intraoperative frozen section biopsies.

\section{Materials and methods}

Study design. The present study retrospectively investigated patients who underwent a surgical resection for pancreatic ductal adenocarcinoma between January 1999 and December 2009 at Yonsei University Health System (Seoul, South Korea). During the study period, a total of 1,119 patients were diagnosed with pancreatic ductal adenocarcinoma and 171 patients $(15.3 \%)$ underwent grossly curative pancreatectomies. Of these patients, 99 with available healthy paraffin-embedded tissue blocks of PALN were re-evaluated using IHC. This study was approved by the ethics committee of Yonsei University Health System (Seoul, Korea).

Surgery and staging. A pancreaticoduodenectomy or distal pancreatectomy with splenectomy was performed, which included a resection of the main pancreatic tumor, the associated regional lymph nodes, the retroperitoneal soft tissue and the PALNs, which allowed for pathological staging. The surgical margins, including the bile duct, pancreatic duct, peripancreatic soft tissue adjacent to the superior mesenteric artery (retroperitoneal margin), duodenum or stomach, were evaluated grossly and microscopically in order to determine their status. These surgical margins, with the exception of the retroperitoneal margin, were analyzed using frozen-sections. If the margin was positive for invasive carcinoma, an additional resection was performed. A pancreatic resection margin without evidence of invasive carcinoma was considered an R0. The final margin status was noted in the permanent pathology report. The TMN stage was evaluated based on the American Joint Committee on Cancer (AJCC) Cancer Staging Manual, 7 th edition (23).

$I H C$. Three serial sectional cuts from formalin-fixed and paraffin-embedded blocks were studied for IHC. Each of the 4- $\mu \mathrm{m}$ sections with $6-\mu \mathrm{m}$ intervals was prepared for IHC staining with CK-19 (M0888 mouse, monoclonal, 1:100; Dako, Copenhagen, Denmark). The IHC was detected using a dextran polymer-based, biotin-free visualization system (Envision kit; Dako). Previous HE-stained sections were re-evaluated for metastasis and the results were compared with the IHC-stained sections by an experienced pathologist. Lymph node micrometastasis was defined as metastatic tumor cells that were detected by IHC evaluation using antibodies against $\mathrm{CK}$, but were missed by routine histological examination using HE staining.

Statistical analysis. The primary goal of the study was to investigate the clinical significance of PALN micrometastasis in resected pancreatic ductal adenocarcinoma. The cumulative survival rates according to overall lymph node metastasis

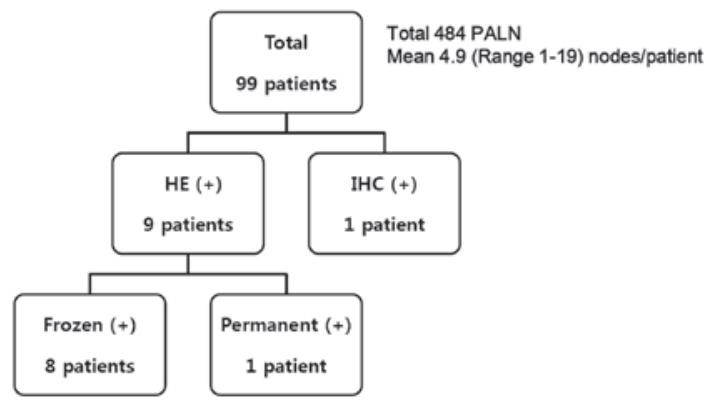

Figure 1. Retrieved PALN assessment in resected pancreatic cancer. A total of 484 PALNs (mean, 4.9 nodes per patient; range, 1-19) were evaluated from the available PALN blocks of 99 patients. Nine patients were identified to exhibit PALN metastasis on routine HE staining (eight patients in frozen sections and one patient in a permanent section). Only one additional patient immunohistochemically demonstrated micrometastasis in a PALN that was not detected otherwise. PALN, para-aortic lymph node; HE, hematoxylin and eosin; IHC, immunohistochemistry.

(pN stage) and PALN metastasis (LN16) were analyzed. The clinicopathological characteristics of the patients with PALN metastasis were summarized. By reviewing the medical records, recurrence and survival data were obtained. Overall survival was defined as the interval between surgery and mortality or the final follow-up visit. The cumulative survival rate was calculated using the Kaplan-Meier method. A log-rank test was used to ascertain the statistically significant differences. $\mathrm{P}<0.05$ was considered to indicate a statistically significant difference.

\section{Results}

Characteristics of patients and resected ductal adenocarcinoma. Among the 99 patients (39 females and 60 males), the mean age was 61.3 years (range, 39-78). Ductal adenocarcinoma was confirmed by microscopic examination in all patients. A total of $21(21.2 \%)$ patients underwent a conventional pancreaticoduodenectomy, 64 (64.6\%) underwent a pylorus-preserving pancreaticoduodenectomy, 13 (13.1\%) underwent a distal pancreatectomy with splenectomy and one (1\%) was treated with a total pancreatectomy. Three patients were diagnosed with pT1 pancreatic cancer, six with pT2, 85 with pT3 and five with pT4. The median tumor size was $2.6 \mathrm{~cm}$ (range, $0.5-8.5$ ). There were no $\mathrm{R} 2$ resections recorded in the medical records. An $\mathrm{R} 1$ resection was performed on 18 patients (18.2\%) and an R0 was performed on 81 patients $(81.8 \%)$.

Retrieved PALN assessment in resected pancreatic cancer. A total of 484 PALNs (mean, 4.9 nodes per patient; range, 1-19) were evaluated from the available PALN blocks in 99 patients (Fig. 1). A total of 13 PALNs (2.7\%) demonstrated metastasis with HE staining in nine patients (eight patients in frozen sections and one patient in a permanent section). All the eight patients who demonstrated PALN metastasis on the HE staining of frozen sections showed a pattern of clustered gland formation and desmoplasia, which occupied the entire involved node (Fig. 2A). The histology of the patient who had PALN metastasis confirmed by HE staining of the permanent section, which was not detected in the frozen section, 
A
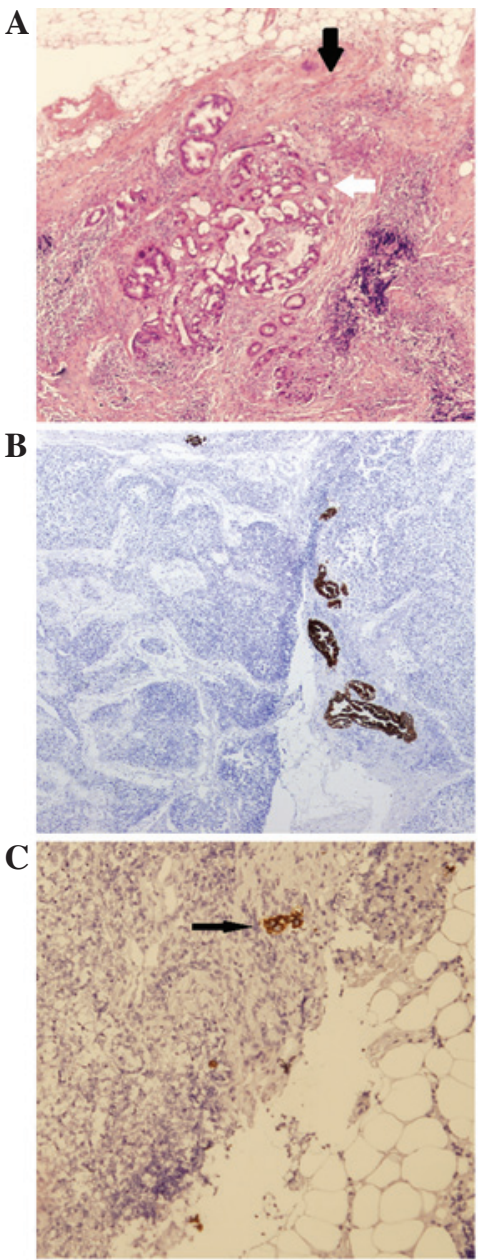

Figure 2. PALN metastasis in pancreatic cancer. (A) PALN metastasis defined in HE staining of frozen biopsy and permanent sections. Note a pattern of clustered gland formation (thick white arrow) and desmoplasia (thick black arrow) in the involved node (HE; magnification, $\mathrm{x} 40$ ). (B) PALN metastasis defined in only HE staining of a permanent section, which was missed in the frozen section, revealed a pattern of scattered small gland formation without desmoplasia. This image of this slide was captured following IHC staining to show the metastatic pattern more precisely (CK-19; magnification, $\mathrm{x} 40$ ). (C) Micrometastasis of PALN demonstrated in IHC staining showing a small size and isolated pattern (thin black arrow; CK-19; magnification, x100). PALN, para-aortic lymph node; HE, hematoxylin and eosin; CK, cytokeratin.

revealed a pattern of scattered small gland formation without desmoplasia (Fig. 2B). IHC reassessment for all 484 PALNs revealed that only one additional patient immunohistochemically demonstrated micrometastasis in a PALN that was not detected otherwise. The CK-19 staining revealed micrometastases in an $\sim 46-\mu \mathrm{m}$ isolated pattern (Fig. $2 \mathrm{C}$ ).

Oncological outcomes of pancreatic cancer with PALN metastasis. The survival rate did not significantly differ based on the $\mathrm{pN}$ staging in the present study. The median survival time was 34 months (95\% CI, 24.3-43.7) for the pN0 tumors and 30 months (95\% CI,: 20.3-39.7) for the $\mathrm{pN} 1$ tumors $(\mathrm{P}=0.223$; Fig. 3A). However, a statistically significant difference was observed in the survival time based on PALN involvement, confirmed by routine HE evaluation. The median survival time was 31 months (95\% CI, 23.9-38.1) in patients without PALN metastases versus 17 months (95\% CI, 12.8-21.6) for patients with PALN metastases ( $\mathrm{P}=0.008$; Fig. $3 \mathrm{~B}$ ).
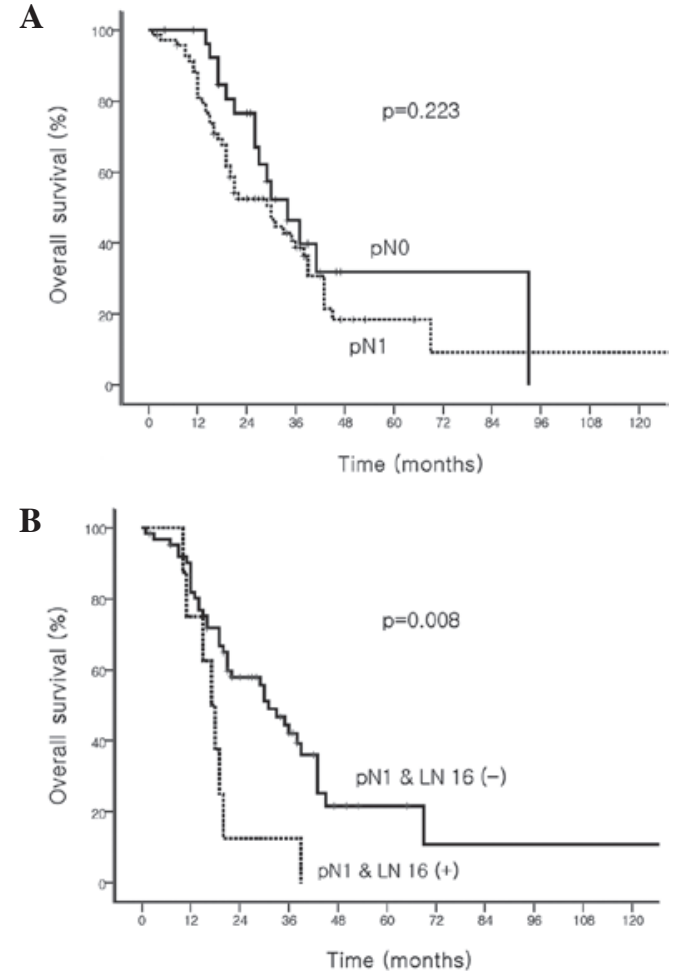

Figure 3. Overall survival according to $\mathrm{pN}$-stage and PALN metastasis in resected ductal adenocarcinoma of the pancreas. (A) No survival difference was noted according to the pN-stage. (B) However, PALN metastasis demonstrated a difference in survival among the patients with pN1 pancreatic cancer. PALN, para-aortic lymph node.

Table I shows the characteristics of the patients with PALN metastasis. Cases 1-8 were the patients who showed PALN metastasis on HE staining of the frozen sections. These patients exhibited large metastatic tumors in the PALNs (median size, $2 \mathrm{~mm}$; range, 0.8-12 $\mathrm{mm}$ ) with clustered gland patterns and extensive desmoplasia. Case 9, who had PALN metastasis confirmed by HE staining of the permanent section, had relatively small metastatic tumors in the PALNs with a pattern of scattered small gland formation and no desmoplasia. Lastly, case 10, who exhibited PALN detected only by IHC staining, possessed extremely small isolated metastatic tumors in the PALNs. All patients who exhibited PALN metastasis confirmed by HE staining (cases 1-9) had other aggressive tumor characteristics, including other regional lymph node metastasis (N1) and lymphovascular and/or perineural invasions, and one patient demonstrated positive resection margins (R1). Case 10 exhibited less aggressive adverse pathological features. The median survival time of cases 1-8 was 17.5 months (range, 10-39). The survival times of cases 9 and 10 were 34 and 41 months, respectively.

\section{Discussion}

Based on the results of the present study, additional IHC using CK-19 antibodies to detect PALN micrometastases in resected pancreatic ductal adenocarcinoma is not an appropriate method to predict prognosis. While the rate of PALN metastasis has been reported to be 6-26\% (24-27), there may be a surgical selection bias that is affecting these numbers. 


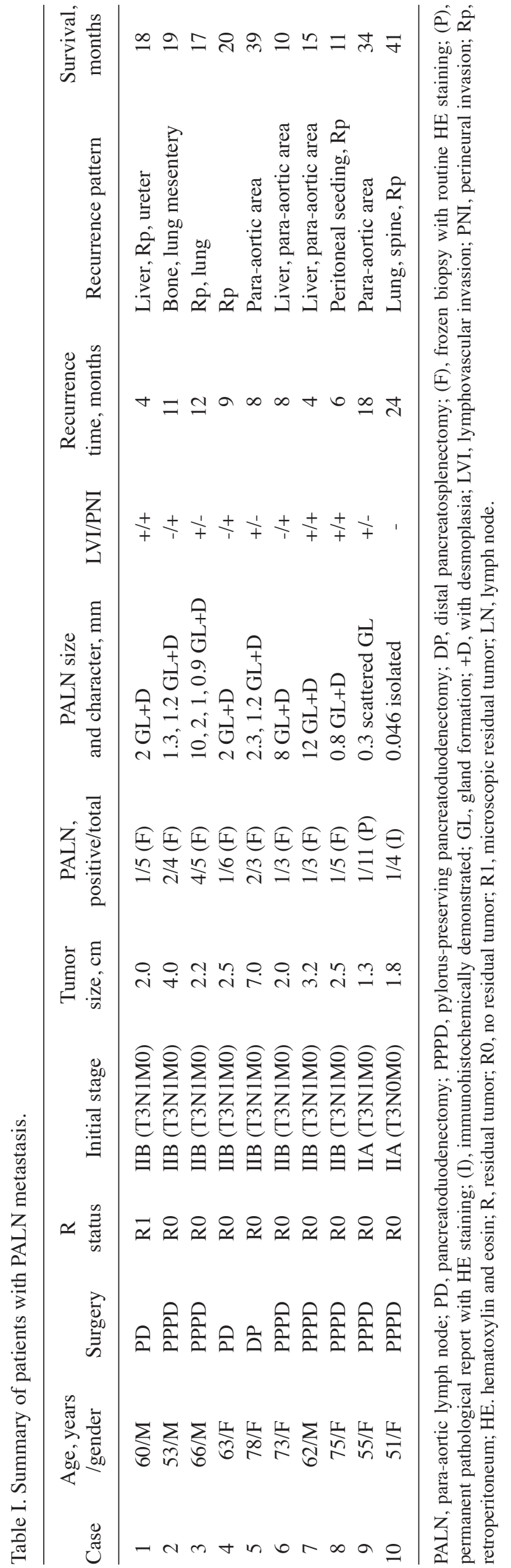

For example, pancreatectomies are generally not performed when PALN metastasis is strongly suggested in pre-operative imaging studies, with observations that include large and conglomerated lymph nodes in the retroperitoneal para-aortic area. As a result, only nine patients (9.1\%) exhibited PALN metastases in routine intraoperative frozen section biopsies and permanent pathological reports, and only one additional patient (1.0\%) was shown to have PALN micrometastasis upon CK-19 staining. Therefore, the incidence of PALN metastasis was observed to be $10.1 \%$. An accurate staging of PALN metastasis conducted by a careful pathological examination with routine HE staining is considered to be sufficient for tumor staging and is useful in predicting prognosis.

Due to the infrequency of resectable pancreatic ductal adenocarcinoma, only a few studies have demonstrated the clinical significance of PALN metastasis in resected pancreatic cancer. Doi et al (20) analyzed the clinicopathological factors in patients with short-term survival who underwent margin-negative radical extended pancreaticoduodenectomy. PALN metastasis was concluded to be the only independent factor for poor prognosis and $\sim 85 \%$ of patients with PALN metastasis succumbed within one year. Shimada et al (28) reported that PALN metastasis was the definitive predictor of recurrence and a shorter survival outcome ( $<12$ months). Yoshida et al (27) also identified the clinicopathological features and surgical outcomes of PALN-positive periampullary adenocarcinoma, and recommended performing intraoperative PALN sampling for frozen section biopsies. The study concluded that radical pancreatectomy with extended soft tissue clearance should not be performed in PALN-positive patients due to a poor oncological outcome. According to the data set of the present study, all eight patients whose frozen section biopsies confirmed PALN metastasis eventually developed tumor recurrences within 12 months of surgery and seven succumbed to the disease within two years (median survival, 17.5 months; Table I). These eight patients had significantly shorter survival periods compared with those patients with regional lymph node metastasis without PALN involvement $(\mathrm{P}<0.008$; Fig. 2B).

Notably, a few cases of long-term survival in patients with PALN metastasis in resected pancreatic cancer have been reported $(21,29)$. Although, as noted previously, studies suggest a poor outcome in PALN-positive resected pancreatic cancer, the majority do not consider the associated characteristics of PALN metastasis. Shimada et al (28), however, observed that PALN metastasis was notably associated with elevated CA 19-9 levels, a larger tumor size and positive surgical margins. Peritoneal cytology was correlated with PALN metastasis in the study $(\mathrm{P}=0.09)$. Furthermore, Yamada, et al (30) concluded that radical surgery may still have value for certain populations of patients with PALN metastasis, such as those aged 60 years or older, patients with tumors of $<4 \mathrm{~cm}$ and those without portal vein involvement. The study also suggested that patients with one lymph node positive for PALN metastasis tended to have an improved prognosis compared with those with two or more positive PALN metastases $(\mathrm{P}=0.14)$.

Although the present data set was rather small and selection bias may have been a factor, it suggests a potential role for curative surgery in certain patient groups with resectable 
pancreatic cancer and PALN involvement. In spite of the poor prognosis in patients who demonstrated PALN metastases observed in routine HE staining, relatively longer survivals were noted in several patients (Table I). Considering a poor median survival time of 5-11 months for unresectable pancreatic tumors without distant metastases (31), the oncological outcomes of the patients included in the present study are thought to be significant, given that curative surgery is the only intervention that may lead to long-term survival in pancreatic cancer. Given the current state of continually improving surgical techniques, perioperative management and adjuvant therapies for pancreatic cancer, the findings of the present study may expand the role of surgery in managing pancreatic cancer, even when PALN metastasis is unexpectedly identified intraoperatively.

According to the present results, all eight patients with PALN metastasis confirmed in frozen section biopsies showed large metastatic tumor sizes and clustered gland patterns with extensive desmoplasia. However, one patient exhibited PALN metastasis that was only identified in routine HE staining of the permanent section, but was missed in the frozen section. This section demonstrated a relatively small size gland pattern without desmoplasia or extensive involvement of the node (Fig. 2). In spite of these histological differences, all of the patients possessed other adverse pathological factors including regional lymph node metastasis, perineural invasion and lymphovascular invasion (Table I). The patient whose PALN metastasis was only detected with IHC (case 10) demonstrated a small, isolated metastasis with less aggressive adverse pathological features. This patient had a relatively long-term survival of 41 months. Notably, despite a small sample size, a correlation was observed between the tumor burden of PALN metastasis and the survival outcome. A small PALN metastasis, which is either undetectable in frozen biopsy or only noticeable in immunostaining, may lead to a longer survival time.

The present study defined lymph node micrometastasis as metastatic tumor cells that are only detectable by IHC staining. However, a universal definition and clinical significance of micrometastasis for pancreatic cancer is still lacking. There are more established characterizations of micrometastasis in breast (32), esophageal $(33,34)$, stomach (35) and colon cancers $(36,37)$ Therefore, the micrometastasis of pancreatic cancer requires further study.

In summary, PALN metastasis that is undetected by routine HE staining in frozen section biopsy, but identified using CK-19 immunostaining, may indicate a relatively lower tumor burden. This may be associated with less aggressive behavior and a favorable prognosis in pancreatic cancer. Therefore, routine HE staining is thought to be sufficient for predicting prognosis. Additionally, in cases where PALN metastasis is unexpectedly identified in intraoperative frozen section biopsies, patients may benefit from curative radical surgery with aggressive adjuvant chemotherapy. However, further large volume investigations are warranted to validate this issue.

\section{Acknowledgements}

This study was supported by the Institute of Gastroenterology, Yonsei University Health System, Seoul, South Korea.

\section{References}

1. Kayahara M, Nagakawa T, Ueno K, Ohta T, Takeda $\mathrm{T}$ and Miyazaki I: An evaluation of radical resection for pancreatic cancer based on the mode of recurrence as determined by autopsy and diagnostic imaging. Cancer 72: 2118-2123, 1993

2. Sener SF, Fremgen A, Menck HR and Winchester DP: Pancreatic cancer: a report of treatment and survival trends for 100,313 patients diagnosed from 1985-1995, using the National Cancer Database. J Am Coll Surg 189: 1-7, 1999.

3. Sperti C, Pasquali C, Piccoli A and Pedrazzoli S: Recurrence after resection for ductal adenocarcinoma of the pancreas. World J Surg 21: 195-200, 1997.

4. Han SS, Jang JY, Kim SW, Kim WH, Lee KU and Park YH: Analysis of long-term survivors after surgical resection for pancreatic cancer. Pancreas 32: 271-275, 2006.

5. Sohn TA, Yeo CJ, Cameron JL, et al: Resected adenocarcinoma of the pancreas-616 patients: results, outcomes, and prognostic indicators. J Gastrointest Surg 4: 567-579, 2000.

6. Howard TJ, Krug JE, Yu J, et al: A margin-negative R0 resection accomplished with minimal postoperative complications is the surgeon's contribution to long-term survival in pancreatic cancer. J Gastrointest Surg 10: 1338-1346, 2006.

7. Raut CP, Tseng JF, Sun CC, et al: Impact of resection status on pattern of failure and survival after pancreaticoduodenectomy for pancreatic adenocarcinoma. Ann Surg 246: 52-60, 2007.

8. Winter JM, Cameron JL, Campbell KA, et al: 1423 pancreaticoduodenectomies for pancreatic cancer: A single-institution experience. J Gastrointest Surg 10: 1199-1211, 2006.

9. Riall TS, Cameron JL, Lillemoe KD, et al: Pancreaticoduodenectomy with or without distal gastrectomy and extended retroperitoneal lymphadenectomy for periampullary adenocarcinoma - part 3: update on 5-year survival. J Gastrointest Surg 9: 1191-1206, 2005.

10. Farnell MB, Pearson RK, Sarr MG, et al; Pancreas Cancer Working Group: A prospective randomized trial comparing standard pancreatoduodenectomy with pancreatoduodenectomy with extended lymphadenectomy in resectable pancreatic head adenocarcinoma. Surgery 138: 618-630, 2005.

11. Pedrazzoli S, DiCarlo V, Dionigi R, et al: Standard versus extended lymphadenectomy associated with pancreatoduodenectomy in the surgical treatment of adenocarcinoma of the head of the pancreas: a multicenter, prospective, randomized study. Lymphadenectomy Study Group. Ann Surg 228: 508-517, 1998 .

12. Iqbal N, Lovegrove RE, Tilney HS, et al: A comparison of pancreaticoduodenectomy with extended pancreaticoduodenectomy: a meta-analysis of 1909 patients. Eur J Surg Oncol 35: 79-86, 2009.

13. Kurahara H, Takao S, Maemura K, et al: Impact of lymph node micrometastasis in patients with pancreatic head cancer. World J Surg 31: 483-492, 2007.

14. Geer RJ and Brennan MF: Prognostic indicators for survival after resection of pancreatic adenocarcinoma. Am J Surg 165: 68-73, 1993.

15. Trede M, Schwall G and Saeger HD: Survival after pancreatoduodenectomy. 118 consecutive resections without an operative mortality. Ann Surg 211: 447-458, 1990.

16. YeoCJ,CameronJL,LillemoeKD, etal:Pancreaticoduodenectomy for cancer of the head of the pancreas. 201 patients. Ann Surg 221: 721-733, 1995.

17. Sobin LH and Wittekind C (eds): TNM Classification of Malignant Tumours (UICC). 5th edition. John Wiley \& Sons, New York, NY, 1997.

18. Japanese Pancreas Society (eds): Classification of Pancreatic Carcinoma. 1st English edition. Kanehara huppan, Tokyo, Japan, 1996.

19. Yoshiaki M, Kenichiro U, Takeshi S, et al: Prognostic impact of para-aortic lymph node metastasis in pancreatic ductal adenocarcinoma. World J Surg 34: 1900-1907, 2010.

20. Doi R, Kami K, Ito D, et al: Prognostic implication of para-aortic lymph node metastasis in resectable pancreatic cancer. World J Surg 31: 147-154, 2007.

21. Sakai M, Nakao A, Kaneko T, et al: Para-aortic lymph node metastasis in carcinoma of the head of the pancreas. Surgery 137: 606-611, 2005.

22. Andersen HB, Baden H, Brahe NE and Burcharth F: Pancreaticoduodenectomy for periampullary adenocarcinoma. J Am Coll Surg 179: 545-552, 1994. 
23. Edge SB, Byrd DR, Compton CC, Fritz AG, Greene FL and Trotti A (eds): AJCC cancer Staging Manual. 7th edition. Springer, New York, NY, 2010.

24. Ishikawa O, Ohigashi H, Sasaki Y, et al: Practical grouping of positive lymph nodes in pancreatic head cancer treated by an extended pancreatectomy. Surgery 121: 244-249, 1997.

25. Nakao A, Harada A, Nonami T, et al: Lymph node metastases in carcinoma of the head of the pancreas region. Br J Surg 82: 399-402, 1995

26. Yoshida T, Aramaki M, Matsumoto T, Morii Y, Sasaki A and Kitano S: The pattern of lymphatic spread in carcinoma of the distal bile duct. Int Surg 83: 124-127, 1998.

27. Yoshida T, Matsumoto T, Sasaki A, Shibata K, Aramaki M and Kitano S: Outcome of paraaortic node-positive pancreatic head and bile duct adenocarcinoma. Am J Surg 187: 736-740, 2004.

28. Shimada K, Sakamoto Y, Sano T and Kosuge T: The role of paraaortic lymph node involvement on early recurrence and survival after macroscopic curative resection with extended lymphadenectomy for pancreatic carcinoma. J Am Coll Surg 203: 345-352, 2006.

29. Kayahara M, Nagakawa T, Ohta T, et al: Analysis of paraaortic lymph node involvement in pancreatic carcinoma: a significant indication for surgery? Cancer 85: 583-590, 1999.

30. Yamada S, Nakao A, Fujii T, et al: Pancreatic cancer with paraaortic lymph node metastasis: a contraindication for radical surgery? Pancreas 38: e13-e17, 2009.
31. Moertel CG, Frytak S, Hahn RG, et al: Therapy of locally unresectable pancreatic carcinoma: a randomized comparison of high dose (6000 rads) radiation alone, moderate dose radiation (4000 rads +5 -fluorouracil), and high dose radiation + 5-fluorouracil: The Gastrointestinal Tumor Study Group. Cancer 48: 1705-1710, 1981

32. McGuckin MA, Cummings MC, Walsh MD, Hohn BG, Bennett IC and Wright RG: Occult axillary node metastases in breast cancer: their detection and prognostic significance. $\mathrm{Br}$ J Cancer 73: 88-95, 1996.

33. Jiao X, Eslami A, Ioffe O, et al: Immunohistochemistry analysis of micrometastasis in pretreatment lymph nodes from patients with esophageal cancer. Ann Thorac Surg 76: 996-1000, 2003.

34. Tanabe T,Nishimaki T, Watanabe H,etal:Immunohistochemically detected micrometastasis in lymph nodes from superficial esophageal squamous cell carcinoma. J Surg Oncol 82: 153-159, 2003.

35. Ishigami S, Natsugoe S, Tokuda K, et al: Clinical impact of micrometastasis of the lymph node in gastric cancer. Am Surg 69: 573-577, 2003.

36. Bilchik AJ, Hoon DS, Saha S, et al: Prognostic impact of micrometastases in colon cancer: interim results of a prospective multicenter trial. Ann Surg 246: 568-577, 2007.

37. Bosch RC: Interview with Dr. Ramón Colomer Bosch, President of the Spanish Society of Medical Oncology (SEOM) by Enrique Garcia Jorda. Clin Transl Oncol 10: 310-312, 2008. 